CASE REPORT

\title{
Cardiac Cysticercosis: A Rare Incidental Finding on Autopsy
}

\author{
Sanjay K, Tapasya S*, Shilpi B, Pansi G, Ritika and Rajeev S \\ Department of Pathology, Pandit Bhagwat Dayal Sharma Post Graduate Institute of Medical Sciences, Rohtak, India
}

*Corresponding author: Tapasya S, MD, Pathologist, Pandit Bhagwat Dayal Sharma Post Graduate Institute of Medical Sciences, Rohtak, India, Tel: 9812694075, E-mail: tapasya.doc@gmail.com

Citation: Sanjay K, Tapasya S, Shilpi B, Pansi G, Ritika, et al. (2017) Cardiac Cysticercosis: A Rare Incidental Finding on Autopsy. J Forensic Crime Stu 1: 107

Article history: Received: 03 November 2017, Accepted: 20 December 2017, Published: 21 December 2017

\begin{abstract}
Cardiac cysticercosis is an extremely rare condition, mostly found in postmortem cases as an incidental finding because it causes either no symptoms or conduction abnormalities in some. We report the case of 40 years male who died of unknown cause. A cystic cavity was seen in heart which on histopathology found to be cysticercosis.
\end{abstract}

Keywords: Cysticercosis; Autopsy

\section{Introduction}

Cysticercosis refers to a parasitic infestation caused by encysted larval stage of the pork tapeworm, Taenia solium. In its life cycle, humans act as definitive host and they harbour the adult forms of the tape worm in their small intestine [1]. Cysticerci can be found anywhere in the body, their location and size determine the clinical presentation.Involvement of heart is very uncommon and mostly asymptomatic [2]. Infection occurs when the tapeworm larvae enter the body. Ingested eggs pass into the bloodstream and disseminate to various organs and form the cysts that characterize cysticercosis [3].

\section{Case Report}

Viscera samples of an adult male aged 40 years in a postmortem case were received. The cause of death was 'unknown' as per postmortem record. The various viscera received for histopathological analysis were brain, heart, lung, liver and kidneys. On gross examination, heart was enlarged, weighed $450 \mathrm{gm}$ and measured 12x8x5 cm. Using the inflow outflow technique the heart

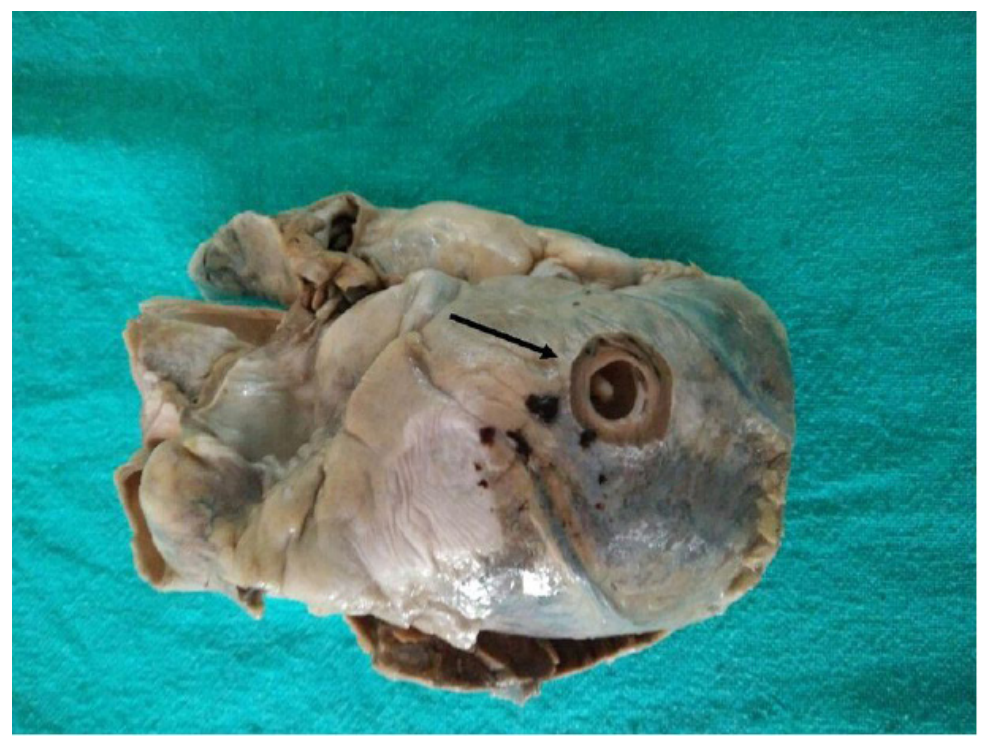

Figure 1: Cystic cavity on the anterior aspect of heart 
was opened. On cutting open, a round well defined cystic cavity measuring $2 \mathrm{~cm}$ diameter was present on the anterior aspect of midline of heart and extending upto the interventricular septum with a depth of $0.4 \mathrm{~cm}$. On opening the four chambers, thickness of right and left ventricular wall measured $0.6 \mathrm{~cm}$ and $1.5 \mathrm{~cm}$ respectively. The stump of aorta measured $2 \mathrm{~cm}$. Interventricular septum was thickened and measured $2.5 \mathrm{~cm}$ and showed a cystic cavity of $2 \mathrm{~cm}$ diameter. The cut surface of the cyst was smooth with well-defined borders and filled with white glistening material within the lumen (Figure 1). Sections from various portions of heart showed larva of cysticercosis in anterior portion of left ventricular wall (Figure 2). Both the coronary arteries and stump of aorta show mild to moderate atherosclerosis. Other organs i.e. brain, lung, liver and kidney were normal in size, weight and morphology. The clinical details of the post-mortem were discussed with family members and it was known that patient had a history of occasional seizures and generalized swelling all over the body since 6 months. No other clinical or investigation details were available. Further sectioning of brain was done but no cyst or any other abnormality was observed.

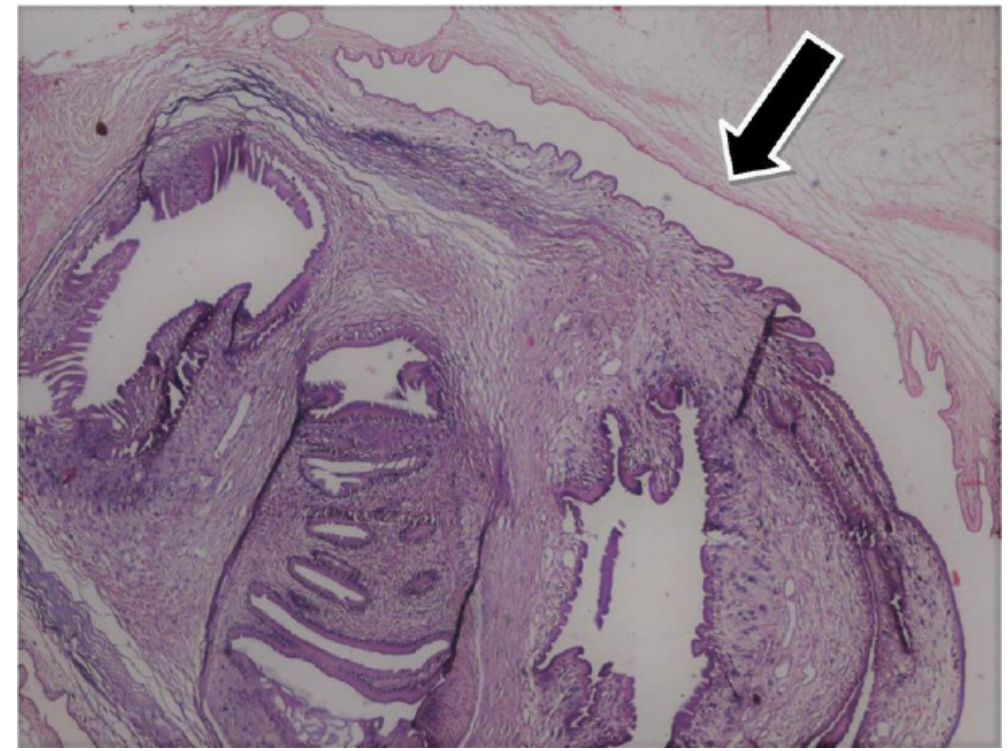

Figure 2: Section from the heart revealing cyst containing larva of cysticercus (H\&E stain 20x)

\section{Discussion}

Cysticercosis is an infection caused by the pork tapeworm, Taenia solium. It is most prevalent in Africa, South-East Asia and South America [3]. Infection occurs via ingestion of contaminated fruits, vegetables and uncooked pork [4]. Larval form disseminate from the intestine via the hepatoportal system to various organs of the body like subcutaneous tissues, skeletal muscles, lungs, brain, eyes, liver and occasionally the heart. Symptoms are variable according to the affected organs like convulsions, decreased visual acuity, arrhythmia, paraparesis, gait abnormality, muscular pseudohypertrophy etc. It is difficult to suspect and diagnose cardiac involvement clinically because of absence of any significant signs and symptoms in most of the cases. Therefore all the patients with disseminated cysticercosis should be screened with echocardiography for any myocardial involvement and carefully monitored during the course of therapy. With the advent and availability of improved imaging modalities to include echocardiography, CT, and MRI, more cases of cardiac cysticercosis are being diagnosed antemortem.

Cardiac cysticercosis is predominantly a silent disease, yet cases in which heart function was adversely affected had been found. Pathological diagnosis of cardiac cysticercosis requires obtaining biopsies from heart which is technically difficult and ethically not justified. Demonstration of cysticerci in the autopsy specimen has been the rule to diagnose involvement of heart in disseminated cysticercosis [2]. Most commonly, it affects the central nervous system. Involvement of heart is extremely rare and is usually a postmortem incidental finding, as evidenced by multiple case reports and autopsy series $[5,6]$. Khelimskll reported the first case in 1962 of cardiac involvement which was detected on autopsy. There was associated skeletal muscle and brain involvement [7]. In India, Reddy and colleagues reported the first case of cysticercosis involving heart [8]. Cysticerci are randomly distributed throughout cardiac tissue, producing a variable inflammatory response ranging from minimal myocardial damage to granuloma formation and potentially fibrosis. Thus, patient is asymptomatic or has conduction abnormalities and arrhythmias $[5,6,9]$. Cysts may be single or multiple, ovoid or round, and range in size from 1.0 to $30.0 \mathrm{~mm}$. They can be randomly distributed in the subpericardium, epicardium, subendocardium, or myocardium of the atria, ventricles, septum, or papillary muscles. Microscopically, a capsule may be visualized with the scolex, hooklets, and suckers underneath. The presence of calcification is variable $[5,9]$.

Parasitic infections previously seen only in developing tropical settings can be currently diagnosed worldwide due to travel and population migration. Treatment of choice is prevention of cysticercosis with a better primary health care system, health education, proper sanitation, better food hygiene, access to safe water and clean water, and eradication of poverty.

\section{References}

1. Baily GG (2003) Cysticercosis. In: Cook GC, Zumla A, editors. Manson's Tropical Disease. (21 ${ }^{\text {st }}$ Edn). London: WB Saunders, $1584-95$. 
2. Bhalla A, Sood A, Sachdeva A (2008) Heart Involvement in Disseminated Cysticercosis: A Case Report and Review of Literature. Indian Heart J 60: $260-2$.

3. Nutman TB, Weller PF (1998) Cestodes. In: Fauci AS, editor. Harrisons Principles of internal medicine. (14 ${ }^{\text {th }}$ Edn). USA: McGraw-Hill, 1225.

4. Murray PR, Rosenthal KS, Pfaller MA (2005) Cestodes. In: Murray PR, editor. Medical Microbiology. (5 ${ }^{\text {th }}$ Edn) USA: Elsevier Mosby 908.

5. Eberly MD, Soh EK, Bannister SP, Tavaf-Motamen H, Scott JS (2008) Isolated cardiac cysticercosis in an adolescent. Pediatr Infect Dis J 27: $369-71$.

6. Bastos AL, Marchiori E, Gasparetto EL, Andrade BH, Junior GC et al. (2007) Pulmonary and cardiac cysticercosis: helical CT findings. Br J Radiol 80: 58-60.

7. Khelimskll AM (1962) Cysticercosis of brain, heart and skeletal muscle. Parazit 31: 610-11.

8. Reddy DJ, Raghavachar V, Majaraj Sgaran B, Vasantha VC (1964) Cysticercosis in Guntur. JIMA 1943: 43.

9. Kirchhoff LV, Weiss LM, Wittner M, Tanowitz HB (2004) Parasitic diseases of the heart. Front Biosci 9: 706-23. 